\title{
Value of FDG PET/CT in the assessment of patients with colon cancer comparing to stand-alone CT
}

\author{
Kaveh Akbari, Franz Fellner, Klaus Emmanuel, Werner Langsteger, Mohsen Beheshti \\ From International Cancer Imaging Society (ICIS) 14th Annual Teaching Course \\ Heidelberg, Germany. 9-11 October 2014
}

\section{Purpose}

To evaluate the potential of FDG PET/CT vs. stand-alone $\mathrm{CT}$ in the assessment of histopathologically verified colon cancer in primary staging, re-staging and follow up.

\section{Material and methods}

70 patients (39 men, 31 women, mean age $70.7 \pm$ 10.7 years) were included in this retrospective study: $28(40 \%)$ primary staging, 28 (40\%) re-staging and 14 (20\%) follow-up patients.

Fifty-eight (58/70) patients $(83 \%)$ had a primary tumour stage of $\geq$ T3. Patients with a known secondary carcinoma were excluded. Diagnostic contrast-enhanced CT was available in all patients (together with PET or in a separate setting with the same acquisition parameters).

The CT and FDG PET reports were examined for all patients. In discordant cases, images of both modalities were re-evaluated by a radiologist and a specialist in nuclear medicine separately.

All results were verified with histological findings or imaging and/or clinical follow-up studies for at least six months.

\section{Results}

In the preoperative setting, additional FDG PET had an influence on the staging in $11(11 / 28)$ patients $(39 \%)$ comparing to $\mathrm{CT}$ alone:

Nine $(9 / 28)$ patients $(32 \%)$ were downstaged, 6 of them with suspicious organ metastases, 3 patients with suspicious lymph node metastases and 1 patient with both suspicious organ metastases and lymph nodes metastases on CT.
Two (2/28) patients (7\%) were upstaged by FDG PET/ $\mathrm{CT}$, one of them with an unclear lung lesion on CT and a malignant hilar lymph node. The second patient showed peritoneal carcinosis on FDG PET.

Comparing with stand-alone CT, only 3 (3/42) patients (7\%) from the restaging and follow-up group were downstaged by additional FDG PET, while concordant findings were seen on both imaging modalities for the rest of the patients.

\section{Conclusion}

This study clearly showed that for primary staging of distant metastases in colon cancer patients FDG PET/CT is more advantageous and overcomes the lower specificity of CT alone.

Comparing both modalities in postoperative cases, FDG PET provides additional findings only in few cases.

Published: 9 October 2014

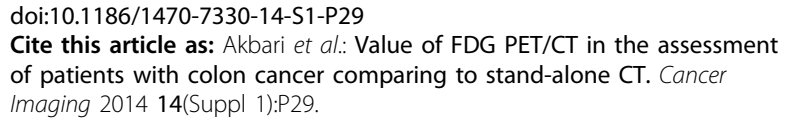

Cite this article as: Akbari et al:: Value of FDG PET/CT in the assessment of patients with colon cancer comparing to stand-alone $\mathrm{CT}$. Cancer Imaging 2014 14(Suppl 1):P29.

* Correspondence: kaveh.akbari@akh.linz.at

Department of Radiology, General Hospital Linz, Austria 